\title{
Effectiveness Of Overuse Injury Prevention Programs On Upper Extremity Performance In Overhead Youth Athletes: A Systematic Review
}

\section{Rachel Lau}

National Institute of Education https://orcid.org/0000-0001-8907-9277

Swarup Mukherjee ( $\square$ swarup.mukherjee@nie.edu.sg )

Nanyang Technological University Singapore https://orcid.org/0000-0001-7626-1299

\section{Research}

Keywords: Youth, Overuse injury, Prevention, Upper extremity, Overhead

Posted Date: July 13th, 2021

DOl: https://doi.org/10.21203/rs.3.rs-685162/v1

License: (c) (i) This work is licensed under a Creative Commons Attribution 4.0 International License.

Read Full License 


\section{Abstract}

\section{Background}

Youth athletes in single hand overhead sport are at risk of upper extremity overuse injuries due to musculoskeletal immaturity, repetitive actions and stress overload. While several upper extremity injury prevention programs have been developed, the effectiveness of upper extremity injury prevention programs on performance outcome measures in overhead youth athletes has not been investigated. This study critically evaluated the effectiveness of existing upper extremity injury prevention programs on performance outcome measures in overhead youth athletes and identified and categorized the training components of these programs.

\section{Methods}

A systematic search was conducted in PubMed, Physiotherapy Evidence Database (PEDro), SPORTDiscus (via EBSCOhost), ScienceDirect, and Web of Science. The inclusion criteria were youth athletes with full participation in throwing or striking sports; the intervention utilized training programs or exercises; control group performed usual training or sham exercises; reported at least one performance outcome measure; and utilized either randomized controlled trials, cluster-randomized controlled trials, or non-randomized controlled trials. Studies had to be published in the English language and within the last two decades. Methodological quality was assessed based on the PEDro Scale.

\section{Results}

Five studies, with a total of 456 youth athletes (age range 10.2-17.1 years) were included. The average PEDro score was 6.6. The effectiveness of the injury prevention programs on the identified performance outcome measures of strength, mobility, and sport-specific measures (ball speed and serve accuracy) were $30.4 \%, 28.6 \%$, and $22.2 \%$, respectively. The training components targeted were categorized as strength, mobility, and plyometrics. Strength was the most common training component and the most widely investigated performance outcome measure among existing upper extremity injury prevention programs.

\section{Conclusion}

Current injury prevention programs have a modest effect on sport performance outcome measures. Future upper extremity injury prevention programs should include training components of strength, mobility and plyometrics in their design given their moderate effects on strength, mobility, and sportspecific outcome measures. Standardized protocols are required for injury prevention program characteristics, and measurement and reporting of performance outcomes measures.

\section{Background}


Youth sports participation has seen an exponential rise over the last few decades. This notion is substantiated by high school athlete participation rate increasing from 5.2 million to 7.9 million over a 30year period [1]. In addition to health benefits and improving social and cognitive skills [2], the growth in youth sport participation is driven by goals akin to those of collegiate athletic scholarship, Olympic dreams, and professional contracts [3]. Moreover, the introduction of youth-centred international sporting mega-events such like the Youth Olympic Games and Asian Youth Games in recent years providing youth athletes with opportunities to excel at a global stage $[4,5]$ can be expected to further encourage youth athletes to engage in competitive sport pursuits.

Increasing youth sport participation is accompanied by a parallel rise in concern for overuse injuries in the youth athlete population [6, 7]. As a part of the process of growth and development, certain unique characteristics such as musculoskeletal and cognitive immaturity renders youth athletes more susceptible to especially overuse injuries compared to their adult counterparts [7-9]. This risk is further magnified with the increasing emphasis on competitive success and the practice of early sport specialization [10]. Youth athletes are subjected to year-round intensive training loads that is associated with a high prevalence of overuse injuries [10-13]. This is an outcome of increased training frequency, excessive stress, repetitive microtrauma to immature musculoskeletal structures, and inadequate recovery periods for tissue adaptation between loading exposures $[7,13]$.

For youth athletes in single-hand overhead sports, there is a growing concern for overuse injuries to the upper extremity [14-16]. This stems from the nature of overhead sports, where the hand is repeatedly raised above the head to repetitively perform a forceful throwing or striking action [17-20]. Moreover, single-hand overhead sports are a popular genre among youths, with four and five overhead sports being amongst the top 10 most popular sports in 2018-19 for boys and girls in the United States, respectively [1]. Examples of these sports include basketball, lacrosse, softball (fast pitch), tennis, and volleyball [1].

The existing literature shows a high prevalence rate of upper extremity overuse injury among youth overhead athletes. In a descriptive epidemiological study on high school female volleyball athletes, $40 \%$ of the athletes reported experiencing shoulder pain that was not associated with a traumatic injury [21]. Another 34-week prospective cohort study on elite junior male handball players [14] reported the average prevalence of shoulder overuse injuries to be $17 \%$ (95\% $\mathrm{Cl}[16-19])$, with a relative burden of $33 \%$ (summed severity score of the shoulder as a proportion of the total severity score of all overuse injuries recorded). Furthermore, surveillance data from the High School Reporting Information Online (RIO) demonstrated that elbow overuse injuries accounted for $24.8 \%$ of all overuse injuries recorded for boy's baseball [22]. Collectively, these studies reflect a high burden magnitude of upper extremity overuse injuries in competitive youth overhead athletes. With decreased performance and growth disturbances identified as consequences of upper extremity overuse injuries [23], there is a clear need for the stakeholders to address these injuries, and their prevention, as a priority.

The overhead motion requires an efficient kinetic chain and strong shoulder structures to allow optimal energy transfer from the lower extremity, through the trunk and the upper extremity, and finally to the hand 
or an implement [18]. Disruption to this mechanism will result in increased stress to the distal segments, increasing the likelihood of an upper extremity overuse injury $[6,8,17,18,24]$. Strong shoulder structures also generate compressive forces of around $1090 \mathrm{~N}$ at the shoulder to counteract the distractive forces acting on the arm during the deceleration phase of the overhead motion[25], which also represents the maximum tensile strength threshold of the soft tissues supporting the shoulder [18]. An overhead youth athlete lacking the necessary fundamental technique to efficiently activate the kinetic chain, and/or adequate strength to withstand the distractive forces from the overhead motion, is at a higher risk of sustaining an upper extremity overuse injury [6]. Therefore, it is critical that corrective and preventive measures are instituted to mitigate these risks in youth athletes.

Various injury prevention programs (IPPs) have been developed and implemented to reduce injury risk among youth athletes $[26,27]$. These IPPs have also been documented in several systematic reviews to be effective at decreasing injury risks in the sample populations [28-30]. However, to enhance our understanding and inform the development of future IPPs, there is a need to identify the components of the program and the underlying mechanisms that may have been effective in decreasing the injury risk [31-33].

Based on the popular 'sequence of prevention' [34], the development of the IPP (and their training components) should be informed by previously identified risk factors of the injury. Moreover, injury prevention and performance enhancement should be considered as parts of the continuum rather than segmented components. Consequently, any reduction in injury risk should be a result of mitigating the identified risk factors, which can be observed via improvements in performance outcome measures such as strength and mobility. In this regard, systematic reviews have been performed to evaluate existing IPPs and their resultant improvements in performance outcome measures among youth athletes. However, the majority of the reviews have focused on lower-extremity IPPs and their respective performance outcome measures [29, 30, 33, 35]. Apparently, no systematic review currently exists that has investigated the effectiveness of upper extremity IPPs on performance outcome measures in the vulnerable population of overhead youth athletes.

The purpose of this systematic review is to evaluate the effectiveness of upper extremity IPPs on performance outcome measures in overhead youth athletes. A secondary aim is to identify and categorize the training components targeted by the existing upper extremity IPPs. The findings from this systematic review will provide greater insights into the planning and development of future IPPs and determine their effectiveness on risk mitigation and sports performance in overhead youth athletes.

\section{Methods}

This systematic review was modelled after the guidelines of the Preferred Reporting Items for Systematic Reviews and Meta-Analyses (PRISMA) [36].

\section{Search Strategy}


An electronic search was conducted using databases of PubMed, Physiotherapy Evidence Database (PEDro), SPORTDiscus (via EBSCOhost), ScienceDirect, and Web of Science. Keywords used in the search included, youth, children, junior, young, adolescent, injury, athletic injury, sports injury, overuse injury, prevention, prevention program, prevention exercises, prevention training, upper extremity, arm, shoulder, elbow, outcomes, outcome measures, performance, performance measures, performance outcomes. Database filters were applied to include studies in English language, published in peer-reviewed academic journals, and within the last two decades to ensure contextual relevance (Year 2000 to 2020). The reference lists of included studies were manually checked for any relevant articles that were not identified during initial database search.

\section{Eligibility criteria}

The inclusion criteria were based on the Population, Intervention, Comparison, Outcome, Study design (PICOS) concept and are as follows: participants were youth athletes with full participation in forceful overhead throwing or striking sports $(P)$, the intervention utilized training programs or exercises for the primary prevention of upper extremity injury (I) with a control group performing usual training or sham exercises (C), at least one performance outcome measure was assessed (0), and the studies utilized randomized controlled trials (RCTs), cluster-RCT, or non-randomised controlled trials (S). Studies were excluded if participants could not participate fully in training and games, were non-overhead sport athletes or college athletes, training programs or exercises were focused on reinjury prevention, interventions were passive in nature (i.e., equipment or legislative changes), had no control group, or only focused on performance enhancement without consideration for injury prevention.

\section{Study selection and data extraction}

Duplicates of articles identified from the search strategy were removed. Titles and abstracts of remaining articles were screened to determine eligibility. Where titles and abstracts of articles were insufficient to confirm eligibility, they were included in the full-text evaluation.

Data extracted from all eligible studies were the authors, the study design, the number of participants, participants' demographics, details of the IPP, performance outcome measures reported, and effectiveness of the IPP on the performance outcome measures.

Based on the details of exercises included in each program, training components were identified to obtain further insights about existing IPPs. As no previous work seemingly exists on the categorisation of training components for upper extremity IPPs, the categories used in this review were adapted from previous work on soccer IPPs [37]. Only three of the six categories of training components, strength, mobility and plyometrics, were relevant and suitable for use for upper extremity IPPs. Notably, an exercise may be categorised under several training components. For example, the 'medicine ball overhead slam' exercise performed in the program by Fernandez-Fernandez et al [38] targets both strength and plyometric components. 
The effectiveness of a program in mitigating intrinsic risk factors was determined by significant improvements in the respective performance outcome measure(s) in the intervention group as compared to the control group [39]. Performance outcome measures were also classified into categories to further understand the measures of interest to researchers. Where data was not reported in the article, corresponding authors were contacted via email.

\section{Assessment of methodological quality}

The methodological quality of the included studies was assessed based on the PEDro Scale [40] and the Newcastle-Ottawa Scale (NOS) [41] for RCT and non-randomised controlled trials, respectively. The maximum score possible on the PEDro scale is 10 , and a score of $\geq 6$ is considered to be of adequate methodological quality [42]. The NOS is based on a star scoring system with a maximum score of 9 , with a score of 6 considered as high quality.

\section{Results}

The initial database search identified 1,111 studies. After removing duplicates and excluding irrelevant studies based on titles and abstracts, 17 studies were identified for full-text screening. Twelve studies were subsequently excluded as they did not meet the eligibility criteria. The reference list of the remaining five studies were screened for potentially suitable studies. One additional study was identified and a total of six studies were selected for inclusion in this review. However, despite the attempts to contact the corresponding author, the data for one study could not be obtained. The study was subsequently dropped from inclusion and ultimately a total of five studies were considered for analysis. Flowchart for study selection is depicted in Fig. 1.

\section{Study characteristics}

A summary of the included studies and their results is presented in Table 1. All studies utilized the RCT design. Both males and females were represented in the studies, although one study [43] did not report the gender of the athletes at the point of group allocation. Sports included were handball, baseball, tennis, and cricket. The number of players in the included studies ranged from 25 to 237 , with a total sample size of 456 for this review. The reported mean age of participants ranged from 10.2 to 17.1 years. 
Table 1

Characteristics of included studies

\begin{tabular}{|c|c|c|c|c|c|c|}
\hline Author/Year & $\begin{array}{l}\text { Study } \\
\text { design }\end{array}$ & Sport & $\begin{array}{l}\text { Participants } \\
\text { (n); Sex; } \\
\text { Age in } \\
\text { years } \\
\text { (mean } \pm \\
\text { SD) }\end{array}$ & $\begin{array}{l}\text { Injury } \\
\text { prevention } \\
\text { program }\end{array}$ & $\begin{array}{l}\text { Upper } \\
\text { extremity } \\
\text { performance } \\
\text { outcome } \\
\text { measures }\end{array}$ & $\begin{array}{l}\text { Effectiveness of } \\
\text { program (IG vs } \\
\text { CG) }\end{array}$ \\
\hline $\begin{array}{l}\text { Fernandez- } \\
\text { Fernandez } \\
\text { et al. [38] }\end{array}$ & RCT & Tennis & $\begin{array}{l}\mathrm{n}=30(\mathrm{IG}= \\
15 ; \mathrm{CG}= \\
15) ; \\
30 \text { males; } \\
14.2 \pm 0.5\end{array}$ & $\begin{array}{l}\text { Strength } \\
\text { training } \\
\text { program }\end{array}$ & $\begin{array}{l}\text { Serve } \\
\text { velocity; } \\
\text { Serve } \\
\text { accuracy }\end{array}$ & $\begin{array}{l}\text { X: Shoulder total } \\
\text { ROM }(p=0.07) ; \\
\text { Serve accuracy } \\
(p=0.95)\end{array}$ \\
\hline $\begin{array}{l}\text { Forrest et } \\
\text { al. [44] }\end{array}$ & RCT & Cricket & $\begin{array}{l}\mathrm{n}=65(\mathrm{IG}= \\
32, \mathrm{CG}= \\
33) ; \\
65 \text { males; } \\
15.6 \pm 1.1\end{array}$ & $\begin{array}{l}\text { Warm-up } \\
\text { program } \\
\text { (adapted } \\
\text { from } \\
\text { Forrest et } \\
\text { al. [70]) }\end{array}$ & $\begin{array}{l}\text { Isokinetic } \\
\text { eccentric } \\
\text { (dominant) } \\
\text { shoulder } \\
\text { external } \\
\text { rotator } \\
\text { strength at } \\
90^{\circ} / \mathrm{s} \text { and } \\
180^{\circ} / \mathrm{s}\end{array}$ & $\begin{array}{l}\text { X: Shoulder } \\
\text { strength at } \\
180^{\circ} / \mathrm{s}(p>0.05)\end{array}$ \\
\hline $\begin{array}{l}\text { Mascarin et } \\
\text { al. [46] }\end{array}$ & RCT & Handball & $\begin{array}{l}\mathrm{n}=25 \text { (IG = } \\
15,8 \\
\text { dominant } \\
\text { hand, } 5 \\
\text { non- } \\
\text { dominant } \\
\text { hand; } C G= \\
10,5 \\
\text { dominant } \\
\text { hand, } 4 \\
\text { non- } \\
\text { dominant } \\
\text { hand); } \\
25 \text { females; } \\
15.5 \pm 0.9\end{array}$ & $\begin{array}{l}\text { Strength } \\
\text { training } \\
\text { program }\end{array}$ & $\begin{array}{l}\text { Dominant } \\
\text { limb } \\
\text { isokinetic } \\
\text { concentric IR } \\
\text { PT at } 60^{\circ} / \mathrm{s} \text {; } \\
\text { Dominant } \\
\text { limb } \\
\text { isokinetic } \\
\text { concentric ER } \\
\text { PT at } 60^{\circ} \% \mathrm{~s} \\
\text { and } 240^{\circ} / \mathrm{s} \text {, } \\
\text { and TW at } \\
60^{\circ} / \mathrm{s} \text {; } \\
\text { Dominant } \\
\text { limb } \\
\text { isokinetic } \\
\text { eccentric ER } \\
\text { PT at } 240^{\circ} / \mathrm{s} \text {; } \\
\text { Non- } \\
\text { dominant } \\
\text { limb }\end{array}$ & $\begin{array}{l}\sqrt{ }: \text { Non- } \\
\text { dominant limb } \\
\text { isokinetic } \\
\text { concentric IR PT } \\
\text { at } 60^{\circ} / \mathrm{s}(p< \\
0.05) ; \text { Non- } \\
\text { dominant limb } \\
\text { isokinetic } \\
\text { concentric ER } \\
\text { PT at } 60^{\circ} / \mathrm{s}(p< \\
0.05) \text { and TW at } \\
60^{\circ} / \mathrm{s}(p<0.05) ; \\
\text { Non-dominant } \\
\text { limb isokinetic } \\
\text { eccentric ER PT } \\
\text { at } 240^{\circ} / \mathrm{s}(p< \\
0.05) ; \text { Dominant } \\
\text { limb shoulder } \\
\text { conventional } \\
\text { strength balance } \\
\text { ratios at } 60^{\circ} / \mathrm{s} \\
(p<0.05)\end{array}$ \\
\hline $\begin{array}{l}C G \text {, control g } \\
\text { rotation; } P T \text {, } \\
\text { significant in } \\
\text { compared to }\end{array}$ & $\begin{array}{l}\text { p; } E R, \epsilon \\
\text { k torqu } \\
\text { ovemer }\end{array}$ & 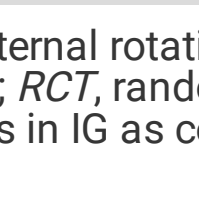 & npared to CG; & non-signific & $\begin{array}{l}\text {, intervention g } \\
\text { רge of motion; } \\
\text { tt improvement }\end{array}$ & 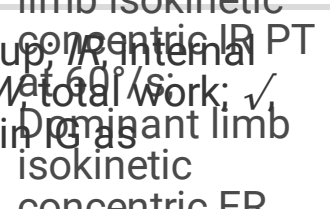 \\
\hline ^Deficits defi & ed as th & 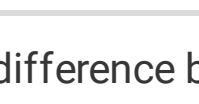 & , & & & $\begin{array}{l}\text { PT at } 60^{\circ} / \mathrm{s} \text { and } \\
240^{\circ} / \mathrm{s} \text {, and TW }\end{array}$ \\
\hline
\end{tabular}




\begin{tabular}{|c|c|c|c|c|c|c|}
\hline Author/Year & $\begin{array}{l}\text { Study } \\
\text { design }\end{array}$ & Sport & $\begin{array}{l}\text { Participants } \\
\text { (n); Sex; } \\
\text { Age in } \\
\text { years } \\
\text { (mean } \pm \\
\text { SD) }\end{array}$ & $\begin{array}{l}\text { Injury } \\
\text { prevention } \\
\text { program }\end{array}$ & $\begin{array}{l}\text { Upper } \\
\text { extremity } \\
\text { performance } \\
\text { outcome } \\
\text { measures }\end{array}$ & 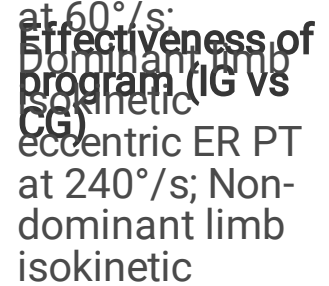 \\
\hline & & & & & $\begin{array}{l}\text { isokinetic } \\
\text { concentric IR } \\
\text { PT at } 60^{\circ} / \mathrm{s} \text {; } \\
\text { Non- } \\
\text { dominant } \\
\text { limb } \\
\text { isokinetic } \\
\text { concentric ER } \\
\text { PT at } 60^{\circ} / \mathrm{s} \\
\text { and } 240^{\circ} / \mathrm{s} \text {, } \\
\text { and TW at } \\
60^{\circ} / \mathrm{s} \text {; } \\
\text { Non- } \\
\text { dominant } \\
\text { limb } \\
\text { isokinetic } \\
\text { eccentric ER } \\
\text { PT at } 240^{\circ} / \mathrm{s} ; \\
\text { Dominant } \\
\text { limb shoulder } \\
\text { strength } \\
\text { balance } \\
\text { ratios } \\
\text { (conventional } \\
\text { ratio at } 60^{\circ} \% \mathrm{~s} \\
\text { and } \\
\text { functional } \\
\text { ratio at } \\
\left.240^{\circ} / \mathrm{s}\right) ; \\
\text { Non- } \\
\text { dominant } \\
\text { limb shoulder } \\
\text { strength } \\
\text { balance } \\
\text { ratios } \\
\text { (conventional } \\
\text { ratio at } 60^{\circ} \% \mathrm{~s} \\
\text { and } \\
\text { functional } \\
\text { ratio at } \\
240^{\circ} / \mathrm{s} \text { ); }\end{array}$ & $\begin{array}{l}\text { concentric ER } \\
\text { PT at } 240^{\circ} / \mathrm{s} ; \\
\text { Dominant limb } \\
\text { shoulder } \\
\text { functional } \\
\text { strength balance } \\
\text { ratios at } 240^{\circ} / \mathrm{s} ; \\
\text { Non-dominant } \\
\text { limb shoulder } \\
\text { strength balance } \\
\text { ratios } \\
\text { (conventional } \\
\text { ratio at } 60^{\circ} / \mathrm{s} \\
\text { and functional } \\
\text { ratio at } 240^{\circ} / \mathrm{s} \text { ); } \\
\text { Dominant limb } \\
\text { ball throwing } \\
\text { velocity } \\
\text { (standing and } \\
\text { jumping); Non- } \\
\text { dominant limb } \\
\text { ball throwing } \\
\text { velocity } \\
\text { (standing and } \\
\text { jumping) }\end{array}$ \\
\hline \multicolumn{7}{|c|}{$\begin{array}{l}C G \text {, control group; } E R \text {, external rotation; } H A \text {, horizontal adduction; } I G \text {, intervention group; } I R \text {, internal } \\
\text { rotation; } P T \text {, peak torque; } R C T \text {, randomized controlled trial; } R O M \text {, range of motion; } T W \text {, total work; } \sqrt{ } \text {, } \\
\text { significant improvements in IG as compared to } C G ; \chi \text {, non-significant improvements in IG as } \\
\text { compared to CG }\end{array}$} \\
\hline
\end{tabular}




\begin{tabular}{|c|c|c|c|c|c|c|}
\hline Author/Year & $\begin{array}{l}\text { Study } \\
\text { design }\end{array}$ & Sport & $\begin{array}{l}\text { Participants } \\
\text { (n); Sex; } \\
\text { Age in } \\
\text { years } \\
\text { (mean } \pm \\
\text { SD) }\end{array}$ & $\begin{array}{l}\text { Injury } \\
\text { prevention } \\
\text { program }\end{array}$ & $\begin{array}{l}\text { Upper } \\
\text { extremity } \\
\text { performance } \\
\text { outcome } \\
\text { measures }\end{array}$ & $\begin{array}{l}\text { Effectiveness of } \\
\text { program (IG vs } \\
\text { CG) }\end{array}$ \\
\hline & & & & & $\begin{array}{l}\text { Dominant } \\
\text { limb ball } \\
\text { throwing } \\
\text { velocity } \\
\text { (standing); }\end{array}$ & \\
\hline & & & & & $\begin{array}{l}\text { Dominant } \\
\text { limb ball } \\
\text { throwing } \\
\text { velocity } \\
\text { (jumping); }\end{array}$ & \\
\hline & & & & & $\begin{array}{l}\text { Non- } \\
\text { dominant } \\
\text { limb ball } \\
\text { throwing } \\
\text { velocity } \\
\text { (standing); }\end{array}$ & \\
\hline & & & & & $\begin{array}{l}\text { Non- } \\
\text { dominant } \\
\text { limb ball } \\
\text { throwing } \\
\text { velocity } \\
\text { (jumping) }\end{array}$ & \\
\hline
\end{tabular}

$C G$, control group; $E R$, external rotation; $H A$, horizontal adduction; $I G$, intervention group; $I R$, internal rotation; $P T$, peak torque; $R C T$, randomized controlled trial; $R O M$, range of motion; $T W$, total work; $\sqrt{ }$, significant improvements in IG as compared to CG; $\chi$, non-significant improvements in IG as compared to CG

${ }^{\wedge}$ Deficits defined as the difference between dominant and non-dominant limbs 


\begin{tabular}{|c|c|c|c|c|c|c|}
\hline Author/Year & $\begin{array}{l}\text { Study } \\
\text { design }\end{array}$ & Sport & $\begin{array}{l}\text { Participants } \\
\text { (n); Sex; } \\
\text { Age in } \\
\text { years } \\
\text { (mean } \pm \\
\text { SD) }\end{array}$ & $\begin{array}{l}\text { Injury } \\
\text { prevention } \\
\text { program }\end{array}$ & $\begin{array}{l}\text { Upper } \\
\text { extremity } \\
\text { performance } \\
\text { outcome } \\
\text { measures }\end{array}$ & $\begin{array}{l}\text { Effectiveness of } \\
\text { program (IG vs } \\
\text { CG) }\end{array}$ \\
\hline $\begin{array}{l}\text { Mascarin et } \\
\text { al. [45] }\end{array}$ & RCT & Handball & $\begin{array}{l}\mathrm{n}=39(\mathrm{IG}= \\
21, \mathrm{CG}= \\
18) ; \\
39 \text { females; } \\
15.2 \pm 1.0\end{array}$ & $\begin{array}{l}\text { Strength } \\
\text { training } \\
\text { program }\end{array}$ & $\begin{array}{l}\text { Isokinetic } \\
\text { concentric IR } \\
\text { PT at } 60^{\circ} / \mathrm{s} \\
\text { and } 240^{\circ} / \mathrm{s} \text {, } \\
\text { and average } \\
\text { power at } \\
240^{\circ} / \mathrm{s} ; \\
\text { Isokinetic } \\
\text { concentric ER } \\
\text { PT at } 60^{\circ} / \mathrm{s} ; \\
\text { Isokinetic } \\
\text { eccentric ER } \\
\text { PT at } 240^{\circ} / \mathrm{s} ; \\
\text { Shoulder } \\
\text { strength } \\
\text { balance } \\
\text { ratios } \\
\text { (conventional } \\
\text { ratio at } 60^{\circ} / \mathrm{s} \\
\text { and } \\
\text { functional } \\
\text { ratio at } \\
240^{\circ} / \mathrm{s} \text { ); } \\
\text { Ball throwing } \\
\text { speed } \\
\text { (standing); } \\
\text { Ball throwing } \\
\text { speed } \\
\text { (jumping) }\end{array}$ & $\begin{array}{l}\sqrt{ } \text { : Isokinetic } \\
\text { concentric IR } \\
\text { average power } \\
\text { at } 240^{\circ} / \mathrm{s}(p= \\
0.05) \\
\chi \text { : Isokinetic } \\
\text { concentric IR PT } \\
\text { at } 60^{\circ} / \mathrm{s}(p= \\
0.87) \text { and } \\
240^{\circ} / \mathrm{s}(p= \\
0.83 \text { ); Isokinetic } \\
\text { concentric ER } \\
\mathrm{PT} \text { at } 60^{\circ} / \mathrm{s} ; \\
\text { Isokinetic } \\
\text { eccentric ER PT } \\
\text { at } 240^{\circ} / \mathrm{s} ; \\
\text { Shoulder } \\
\text { strength balance } \\
\text { ratios } \\
\text { (conventional } \\
\text { ratio at } 60^{\circ} / \mathrm{s} \\
\text { and functional } \\
\text { ratio at } 240^{\circ} / \mathrm{s} \text { ); } \\
\text { Ball throwing } \\
\text { speed } \\
\text { (standing); Ball } \\
\text { throwing speed } \\
\text { (jumping) }\end{array}$ \\
\hline
\end{tabular}

$C G$, control group; $E R$, external rotation; $H A$, horizontal adduction; $I G$, intervention group; $I R$, internal rotation; $P T$, peak torque; $R C T$, randomized controlled trial; $R O M$, range of motion; $T W$, total work; $\sqrt{ }$, significant improvements in IG as compared to CG; $\chi$, non-significant improvements in IG as compared to CG

${ }^{\wedge}$ Deficits defined as the difference between dominant and non-dominant limbs 


\begin{tabular}{|c|c|c|c|c|c|c|}
\hline Author/Year & $\begin{array}{l}\text { Study } \\
\text { design }\end{array}$ & Sport & $\begin{array}{l}\text { Participants } \\
\text { (n); Sex; } \\
\text { Age in } \\
\text { years } \\
\text { (mean } \pm \\
\text { SD) }\end{array}$ & $\begin{array}{l}\text { Injury } \\
\text { prevention } \\
\text { program }\end{array}$ & $\begin{array}{l}\text { Upper } \\
\text { extremity } \\
\text { performance } \\
\text { outcome } \\
\text { measures }\end{array}$ & $\begin{array}{l}\text { Effectiveness of } \\
\text { program (IG vs } \\
\text { CG) }\end{array}$ \\
\hline $\begin{array}{l}\text { Sakata et } \\
\text { al. [43] }\end{array}$ & RCT & Baseball & $\begin{array}{l}\mathrm{n}=237(\mathrm{IG} \\
=117, \mathrm{CG}= \\
120) ; \\
\text { sex not } \\
\text { stated; } \\
10.2 \pm 0.8\end{array}$ & $\begin{array}{l}\text { Modified } \\
\text { Yokohama } \\
\text { Baseball-9 } \\
\text { (mYKB-9) }\end{array}$ & $\begin{array}{l}\text { Ball speed; } \\
\text { Elbow } \\
\text { extension } \\
\text { deficits } \\
\text { ROM of } \\
\text { shoulder ER } \\
\text { deficits }{ }^{\wedge} \text { in } \\
90^{\circ} \text { of } \\
\text { abduction; } \\
\text { ROM of } \\
\text { shoulder IR } \\
\text { deficits in } \\
90^{\circ} \text { of } \\
\text { abduction; } \\
\text { Total } \\
\text { shoulder } \\
\text { ROM in } 90^{\circ} \\
\text { of abduction; } \\
\text { ROM of } \\
\text { shoulder HA } \\
\text { deficits } \\
\text { Thoracic } \\
\text { kyphosis } \\
\text { angle }\end{array}$ & $\begin{array}{l}\sqrt{ } \text { : Ball speed ( } p \\
=0.01) ; \text { ROM of } \\
\text { shoulder HA } \\
\text { deficits }{ }^{\wedge}(p= \\
0.03) ; \text { Thoracic } \\
\text { kyphosis angle } \\
(p=0.03) \\
\\
\text { x: Elbow } \\
\text { extension } \\
\text { deficits } ; \text { ROM of } \\
\text { shoulder ER } \\
\text { deficits in } 90^{\circ} \\
\text { of abduction; } \\
\text { ROM of shoulder } \\
\text { IR deficits^ in } \\
90^{\circ} \text { of } \\
\text { abduction; Total } \\
\text { shoulder ROM in } \\
90^{\circ} \text { of abduction }\end{array}$ \\
\hline $\begin{array}{l}C G \text {, control gr } \\
\text { rotation; } P T, \mathrm{p} \\
\text { significant im } \\
\text { compared to }\end{array}$ & $\begin{array}{l}\text { up; } E R, \mathrm{e} \\
\text { ak torqu } \\
\text { rovemen } \\
\mathrm{G}\end{array}$ & $\begin{array}{l}\text { ernal rotat } \\
R C T \text {, rand } \\
\text { in IG as c }\end{array}$ & $\begin{array}{l}\text {; } H A \text {, horizon } \\
\text { ized controll } \\
\text { ppared to } C G ;\end{array}$ & $\begin{array}{l}\text { adduction; } \\
\text { trial; } R O M \text {, } \\
\text { non-signific }\end{array}$ & $\begin{array}{l}\text { intervention g } \\
\text { ige of motion; }\end{array}$ & $\begin{array}{l}\text { up; IR, internal } \\
V, \text { total work; } \sqrt{ } \text {, } \\
\text { in IG as }\end{array}$ \\
\hline
\end{tabular}

\section{Methodological quality assessment}

The methodological quality of the five included studies is presented in Table 2. As all studies were RCTs, only the PEDro scale was utilized. The average score was 6.6 , with a highest and lowest score of 8 [43] and 5 [44] respectively. The three common methodological deficits identified by the PEDro scale was the failure to blind all subjects, all therapists (who administered the therapy), and all assessors (who measured at least one key outcome). 
Table 2

Methodological quality assessment using the PEDro scale

\begin{tabular}{|c|c|c|c|c|c|c|}
\hline $\begin{array}{l}\text { Item } \\
\text { No. }\end{array}$ & Item & $\begin{array}{l}\text { Fernandez- } \\
\text { Fernandez } \\
\text { et al. [38] }\end{array}$ & $\begin{array}{l}\text { Forrest } \\
\text { et al. } \\
\text { [44] }\end{array}$ & $\begin{array}{l}\text { Mascarin } \\
\text { et al. [46] }\end{array}$ & $\begin{array}{l}\text { Mascarin } \\
\text { et al. [45] }\end{array}$ & $\begin{array}{l}\text { Sakata } \\
\text { et al. } \\
\text { [43] }\end{array}$ \\
\hline 1 & Eligibility criteria were specified & Y & Y & Y & Y & Y \\
\hline 2 & $\begin{array}{l}\text { Subjects were randomly } \\
\text { allocated to groups }\end{array}$ & 1 & 1 & 1 & 1 & 1 \\
\hline 3 & Allocation was concealed & 0 & 0 & 1 & 1 & 1 \\
\hline 4 & $\begin{array}{l}\text { The groups were similar at } \\
\text { baseline regarding the most } \\
\text { important prognostic indicators }\end{array}$ & 1 & 0 & 1 & 1 & 1 \\
\hline 5 & $\begin{array}{l}\text { There was blinding of all } \\
\text { subjects }\end{array}$ & 0 & 0 & 0 & 0 & 0 \\
\hline 6 & $\begin{array}{l}\text { There was blinding of all } \\
\text { therapists who administered the } \\
\text { therapy }\end{array}$ & 0 & 0 & 0 & 0 & 0 \\
\hline 7 & $\begin{array}{l}\text { There was blinding of all } \\
\text { assessors who measured at } \\
\text { least one key outcome }\end{array}$ & 0 & 0 & 0 & 0 & 1 \\
\hline 8 & $\begin{array}{l}\text { Measures of at least one key } \\
\text { outcome were obtained from } \\
\text { more than } 85 \% \text { of the subjects } \\
\text { initially allocated to groups }\end{array}$ & 1 & 1 & 1 & 1 & 1 \\
\hline 9 & $\begin{array}{l}\text { All subjects for whom outcome } \\
\text { measures were available } \\
\text { received the treatment or control } \\
\text { condition as allocated or, where } \\
\text { this was not the case, data for at } \\
\text { least one key outcome was } \\
\text { analyzed by "intention to treat" }\end{array}$ & 1 & 1 & 1 & 1 & 1 \\
\hline 10 & $\begin{array}{l}\text { The results of between-group } \\
\text { statistical comparisons are } \\
\text { reported for at least one key } \\
\text { outcome }\end{array}$ & 1 & 1 & 1 & 1 & 1 \\
\hline 11 & $\begin{array}{l}\text { The study provides both point } \\
\text { measures and measures of } \\
\text { variability for at least one key } \\
\text { outcome }\end{array}$ & 1 & 1 & 1 & 1 & 1 \\
\hline & Total PEDro score & 6 & 5 & 7 & 7 & 8 \\
\hline
\end{tabular}

Types of injury prevention programs 
Three out of five studies identified their IPPs to be strength training programs [38, 45, 46]. One study utilized the modified Yokohama Baseball-9 (mYKB-9) program [43] which consisted of stretching, dynamic mobility, and lower extremity balance. The mYKB-9 is an improved version of the original Yokohama Baseball-9 (YKB-9) program [47]. The final study in this review identified their program as an exercise-based IPP that was performed as an alternative to normal warm-up for training sessions [44]. Therefore, it is considered as a warm-up exercise program for this review.

\section{Training components}

Overall, three training components were targeted by the five IPPs - strength, mobility, and plyometrics. The most common training component targeted was strength (4/5 studies). Mobility was targeted as a training component in only one IPP. Similarly, plyometrics was also targeted in only one IPP. Detailed information about the exercises and targeted training components of each IPP for the upper extremity is presented in Table 3. 
Table 3

Exercises and targeted training components for the upper extremity in each injury prevention program

\begin{tabular}{|c|c|c|c|c|c|c|}
\hline Study & Intervention & $\begin{array}{l}\text { Details of } \\
\text { injury } \\
\text { prevention } \\
\text { program }^{+}\end{array}$ & $\begin{array}{l}\text { All exercises in } \\
\text { the program }\end{array}$ & $\begin{array}{l}\text { Mobility } \\
\text { (ROM) of } \\
\text { the } \\
\text { upper } \\
\text { extremity }\end{array}$ & $\begin{array}{l}\text { Plyometric } \\
\text { of the } \\
\text { upper } \\
\text { extremity }\end{array}$ & $\begin{array}{l}\text { Strength } \\
\text { of the } \\
\text { upper } \\
\text { extremity }\end{array}$ \\
\hline \multirow[t]{3}{*}{$\begin{array}{l}\text { Fernandez- } \\
\text { Fernandez } \\
\text { et al. [38] }\end{array}$} & \multirow[t]{3}{*}{$\begin{array}{l}\text { Strength } \\
\text { training } \\
\text { program }\end{array}$} & \multirow{3}{*}{$\begin{array}{l}3 \times p e r \\
\text { week, (60 } \\
\text { to } 70 \\
\text { minutes); } \\
6 \text { weeks; } \\
\text { Equipment } \\
\text { required: } \\
\text { Elastic } \\
\text { tubing and } \\
\text { medicine } \\
\text { ball }\end{array}$} & $\begin{array}{l}\text { Core: crunches, } \\
\text { reverse crunches, } \\
\text { oblique crunches, } \\
\text { plank, side plank }\end{array}$ & \multirow[t]{3}{*}{$\begin{array}{l}\text { Not } \\
\text { included }\end{array}$} & \multirow[t]{3}{*}{ Included } & \multirow[t]{3}{*}{ Included } \\
\hline & & & $\begin{array}{l}\text { Elastic tubing: } \\
\text { triceps (elbow } \\
\text { extension), } \\
\text { rowing, external } \\
\text { rotation with } \\
\text { shoulder flexed } \\
90^{\circ} \text {, external } \\
\text { rotation with } \\
\text { shoulder } \\
\text { abducted } 90^{\circ}, \\
\text { shoulder } \\
\text { abduction to } 90^{\circ} \text {, } \\
\text { diagonal pattern } \\
\text { flexion, reverse } \\
\text { throw, standard } \\
\text { forward throw, } \\
\text { wrist flexion- } \\
\text { extension }\end{array}$ & & & \\
\hline & & & $\begin{array}{l}\text { Medicine ball: } \\
\text { chest pass, } \\
\text { overhead throw, } \\
\text { ear throw, squat } \\
\text { to thrust, } \\
\text { overhead slam, } \\
\text { diagonal wood- } \\
\text { chop, close- } \\
\text { stance throw }\end{array}$ & & & \\
\hline $\begin{array}{l}\text { Forrest et } \\
\text { al. [44] }\end{array}$ & $\begin{array}{l}\text { Warm-up } \\
\text { exercise } \\
\text { program }\end{array}$ & $\begin{array}{l}2 \times \text { per } \\
\text { week (10 } \\
\text { to } 15 \\
\text { minutes); } \\
8 \text { weeks; } \\
\text { Equipment } \\
\text { required: } \\
\text { Cricket } \\
\text { balls and } \\
\text { resistance } \\
\text { bands }\end{array}$ & $\begin{array}{l}\text { Dynamic warm } \\
\text { up, shoulder } \\
\text { external rotation } \\
\text { strengthening, hip } \\
\text { adductor } \\
\text { strengthening, } \\
\text { Nordic hamstring } \\
\text { strengthening, } \\
\text { single-leg ball } \\
\text { throw, squats, } \\
\text { lunges, prone-hold }\end{array}$ & $\begin{array}{l}\text { Not } \\
\text { included }\end{array}$ & $\begin{array}{l}\text { Not } \\
\text { included }\end{array}$ & Included \\
\hline
\end{tabular}

mYKB-9, modified Yokohama Baseball-9

${ }^{+}$Frequency (duration of program during training, if applicable), length of study, and equipment used. 


\begin{tabular}{|c|c|c|c|c|c|c|}
\hline Study & Intervention & $\begin{array}{l}\text { Details of } \\
\text { injury } \\
\text { prevention } \\
\text { program }^{+}\end{array}$ & $\begin{array}{l}\text { All exercises in } \\
\text { the program }\end{array}$ & $\begin{array}{l}\text { Mobility } \\
\text { (ROM) of } \\
\text { the } \\
\text { upper } \\
\text { extremity }\end{array}$ & $\begin{array}{l}\text { Plyometric } \\
\text { of the } \\
\text { upper } \\
\text { extremity }\end{array}$ & $\begin{array}{l}\text { Strength } \\
\text { of the } \\
\text { upper } \\
\text { extremity }\end{array}$ \\
\hline $\begin{array}{l}\text { Mascarin } \\
\text { et al. [46] }\end{array}$ & $\begin{array}{l}\text { Strength } \\
\text { training } \\
\text { program }\end{array}$ & $\begin{array}{l}3 \times \text { per } \\
\text { week; } 6 \\
\text { weeks; } \\
\text { Equipment } \\
\text { required: } \\
\text { Resistance } \\
\text { bands }\end{array}$ & $\begin{array}{l}\text { Shoulder external } \\
\text { rotation } \\
\text { strengthening in } \\
\text { (1) standing } \\
\text { position with } 90^{\circ} \\
\text { shoulder } \\
\text { abduction and } \\
90^{\circ} \text { elbow flexion } \\
\text { and (2) standing } \\
\text { position with } \\
\text { shoulder in } \\
\text { neutral position } \\
\text { and elbow flexed } \\
\text { at } 90^{\circ}\end{array}$ & $\begin{array}{l}\text { Not } \\
\text { included }\end{array}$ & $\begin{array}{l}\text { Not } \\
\text { included }\end{array}$ & Included \\
\hline $\begin{array}{l}\text { Mascarin } \\
\text { et al. [45] }\end{array}$ & $\begin{array}{l}\text { Strength } \\
\text { training } \\
\text { program }\end{array}$ & $\begin{array}{l}3 \times \text { per } \\
\text { week; } 6 \\
\text { weeks; } \\
\text { Equipment } \\
\text { required: } \\
\text { Resistance } \\
\text { bands }\end{array}$ & $\begin{array}{l}\text { Shoulder internal } \\
\text { rotation } \\
\text { strengthening in } \\
\text { (2) standing } \\
\text { position with } 90^{\circ} \\
\text { shoulder } \\
\text { abduction and } \\
90^{\circ} \text { elbow flexion } \\
\text { and (2) standing } \\
\text { position with } \\
\text { shoulder in } \\
\text { neutral position } \\
\text { and elbow flexed } \\
\text { at } 90^{\circ}\end{array}$ & $\begin{array}{l}\text { Not } \\
\text { included }\end{array}$ & $\begin{array}{l}\text { Not } \\
\text { included }\end{array}$ & Included \\
\hline
\end{tabular}

mYKB-9, modified Yokohama Baseball-9

${ }^{+}$Frequency (duration of program during training, if applicable), length of study, and equipment used. 


\begin{tabular}{|c|c|c|c|c|c|c|}
\hline Study & Intervention & $\begin{array}{l}\text { Details of } \\
\text { injury } \\
\text { prevention } \\
\text { program }^{+}\end{array}$ & $\begin{array}{l}\text { All exercises in } \\
\text { the program }\end{array}$ & $\begin{array}{l}\text { Mobility } \\
\text { (ROM) of } \\
\text { the } \\
\text { upper } \\
\text { extremity }\end{array}$ & $\begin{array}{l}\text { Plyometric } \\
\text { of the } \\
\text { upper } \\
\text { extremity }\end{array}$ & $\begin{array}{l}\text { Strength } \\
\text { of the } \\
\text { upper } \\
\text { extremity }\end{array}$ \\
\hline $\begin{array}{l}\text { Sakata et } \\
\text { al. [43] }\end{array}$ & $\begin{array}{l}\text { Modified } \\
\text { Yokohama } \\
\text { Baseball-9 } \\
\text { (mYKB-9) }\end{array}$ & $\begin{array}{l}\text { At least } 1 \times \\
\text { per week } \\
\text { (10 } \\
\text { minutes); } \\
12 \\
\text { months; } \\
\text { Equipment } \\
\text { required: } \\
\text { none }\end{array}$ & $\begin{array}{l}\text { Stretching: } \\
\text { massage of } \\
\text { brachial muscles, } \\
\text { stretch of } \\
\text { pronator muscles, } \\
\text { posterior shoulder } \\
\text { stretch, anterior } \\
\text { shoulder stretch, } \\
\text { posterior hip } \\
\text { stretch } \\
\text { Dynamic mobility: } \\
\text { cat and dog } \\
\text { exercise, trunk } \\
\text { rotation exercise } \\
\\
\text { Lower extremity } \\
\text { balance: lateral } \\
\text { slide exercise, } \\
\text { elbow-to-knee } \\
\text { exercise }\end{array}$ & Included & $\begin{array}{l}\text { Not } \\
\text { included }\end{array}$ & $\begin{array}{l}\text { Not } \\
\text { included }\end{array}$ \\
\hline mYKB-9, m & ied Yokohan & Baseball-9 & & & & \\
\hline
\end{tabular}

\section{Effect of injury prevention programs on performance outcome measures}

The breakdown of the upper extremity performance outcome measures into the categories of strength, mobility and sport-specific outcome measures are presented in Table 4. The overall effectiveness of the identified IPPs on performance outcome measures are presented in Table 5. 
Table 4

Upper extremity performance outcome measures categorised by performance category

\section{Performance category}

Study

Upper extremity performance outcome measures
Combined list of upper extremity performance outcome measures

Strength

1) Isokinetic
Isokinetic eccentric (D) external rotator strength at $90 \%$ and $180^{\circ} / \mathrm{s}$ al. [44]

Mascarin et al. [46]

D isokinetic concentric IR PT at $60 \%$ s; D isokinetic concentric ER PT at $60 \%$ s and $240 \%$, and TW at $60 \%$; D isokinetic eccentric ER PT at $240 \%$ s;

ND isokinetic concentric IR PT at $60 \%$ s; ND isokinetic concentric ER PT at $60 \%$ s and $240 \%$, and TW at $60 \%$; ND isokinetic eccentric ER PT at $240 \%$;

$D$ isokinetic strength balance ratios (conventional ratio at $60 \%$ and functional ratio at $240 \%$ s);

ND strength balance ratios (conventional ratio at $60 \%$ s and functional ratio at $240 \%$ s)

Mascarin Isokinetic concentric IR PT at $60 \%$ s and $240 \%$, et al. [45] and average power at $240 \%$ s;

Isokinetic concentric ER PT at $60^{\circ} / \mathrm{s}$;

Isokinetic eccentric ER PT at $240 \%$ s;

Strength balance ratios (conventional ratio at $60 \%$ s and functional ratio at $240 \%$ s) concentric (conventional) ER/IR strength ratio at $60 \% \mathrm{~s}(\mathrm{D})$ $[\times 2]$

2) Isokinetic concentric IR PT at $60 \%$ (D) [x2]

3) Isokinetic concentric ER PT at $60 \%$ (D) [×2]

4) Isokinetic eccentric ER PT at $240 \%$ s (D) [×2]

5) Isokinetic functional ER/IR ratio at $240 \%$ (D) [×2]

6) Isokinetic eccentric ER strength at $90 \% \mathrm{~s}$

7) Isokinetic eccentric ER strength at $180 \% \mathrm{~s}$

8) Isokinetic concentric ER PT at $240^{\circ} / \mathrm{s}$ (D)

9) Isokinetic concentric ER PT at $240^{\circ} / \mathrm{s}$ (ND)

10) Isokinetic concentric ER TW at $60 \%$ s (D)

11) Isokinetic concentric ER TW

D, dominant shoulder; ND, non-dominant shoulder; ER, external rotation; HA, horizontal adduction; IR, internal rotation; PT, peak torque; ROM, range of motion; TW, total work

${ }^{\wedge}$ Deficits defined as the difference between dominant and non-dominant limbs 


\begin{tabular}{|c|c|c|c|}
\hline $\begin{array}{l}\text { Performance } \\
\text { category }\end{array}$ & Study & $\begin{array}{l}\text { Upper extremity performance outcome } \\
\text { measures }\end{array}$ & $\begin{array}{l}\text { Combined list of } \\
\text { upper extremity } \\
\text { performance } \\
\text { outcome measures }\end{array}$ \\
\hline & & & at $60^{\circ} / \mathrm{s}(\mathrm{ND})$ \\
\hline & & & $\begin{array}{l}\text { 12) Isokinetic } \\
\text { concentric IR PT at } \\
60^{\circ} / \mathrm{s} \text { (ND) }\end{array}$ \\
\hline & & & $\begin{array}{l}\text { 13) Isokinetic } \\
\text { concentric ER PT } \\
\text { at } 60^{\circ} / \mathrm{s}(\mathrm{ND})\end{array}$ \\
\hline & & & $\begin{array}{l}\text { 14) Isokinetic } \\
\text { eccentric ER PT at } \\
240^{\circ} / \mathrm{s} \text { (ND) }\end{array}$ \\
\hline & & & $\begin{array}{l}\text { 15) Isokinetic } \\
\text { concentric } \\
\text { (conventional) } \\
\text { ER/IR strength } \\
\text { ratio at } 60^{\circ} / \mathrm{s}(\mathrm{ND})\end{array}$ \\
\hline & & & $\begin{array}{l}\text { 16) Isokinetic } \\
\text { concentric IR PT at } \\
240^{\circ} / \mathrm{s}\end{array}$ \\
\hline & & & $\begin{array}{l}\text { 17) Isokinetic } \\
\text { concentric IR } \\
\text { average power at } \\
240^{\circ} / \mathrm{s}\end{array}$ \\
\hline & & & $\begin{array}{l}\text { 18) Isokinetic } \\
\text { functional ER/IR } \\
\text { strength ratio at } \\
240^{\circ} / \mathrm{s}(\mathrm{ND})\end{array}$ \\
\hline \multirow[t]{4}{*}{$\begin{array}{l}\text { Mobility } \\
\text { (ROM) }\end{array}$} & \multirow[t]{4}{*}{$\begin{array}{l}\text { Fernandez- } \\
\text { Fernandez } \\
\text { et al. [38] }\end{array}$} & \multirow[t]{4}{*}{ Total shoulder ROM } & $\begin{array}{l}\text { 1) Total ROM (D) } \\
{[\times 2]}\end{array}$ \\
\hline & & & $\begin{array}{l}\text { 2) Elbow extension } \\
\text { deficits }\end{array}$ \\
\hline & & & $\begin{array}{l}\text { 3) ROM of } \\
\text { shoulder ER } \\
\text { deficits^ in } 90^{\circ} \text { of } \\
\text { abduction }\end{array}$ \\
\hline & & & $\begin{array}{l}\text { 4) ROM of } \\
\text { shoulder IR } \\
\text { deficits in } 90^{\circ} \text { of } \\
\text { abduction }\end{array}$ \\
\hline \multicolumn{4}{|c|}{ 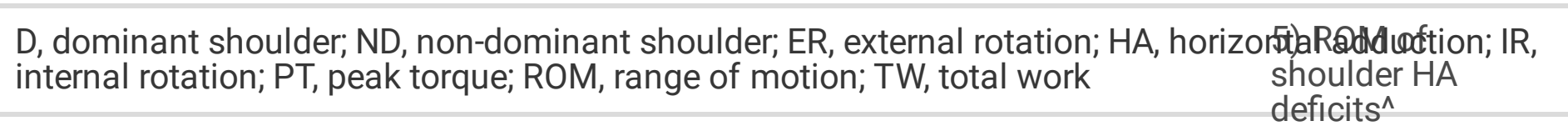 } \\
\hline
\end{tabular}




\begin{tabular}{|c|c|c|c|}
\hline $\begin{array}{l}\text { Performance } \\
\text { category }\end{array}$ & Study & $\begin{array}{l}\text { Upper extremity performance outcome } \\
\text { measures }\end{array}$ & $\begin{array}{l}\text { Eombingedigist of } \\
\text { upper extremity } \\
\text { performance } \\
\text { outcome measures }\end{array}$ \\
\hline
\end{tabular}

Sakata et Elbow extension deficits ${ }^{\wedge}$; ROM of shoulder ER al. [43] deficits in $90^{\circ}$ of abduction; ROM of shoulder IR deficits in $90^{\circ}$ of abduction; Total shoulder ROM in $90^{\circ}$ of abduction; ROM of shoulder HA deficits; Thoracic kyphosis angle

$\begin{array}{llll}\text { Sport- } & \text { Fernandez- } & \text { Serve velocity; Serve accuracy } & \text { 1) Serve velocity }\end{array}$ specific Fernandez

et al. [38]

/Throwing velocity (standing)/ Throwing speed (standing)/Ball speed (D) [x4]
Mascarin
D ball throwing velocity standing and jumping;
et al. [46]
ND ball throwing velocity standing and jumping

2) Throwing velocity jumping/Throwing speed jumping (D) [×2]

$\begin{array}{ll}\text { Mascarin } & \text { Ball throwing speed (standing); Ball throwing } \\ \text { et al. [45] } & \begin{array}{l}\text { speed (jumping) }\end{array}\end{array}$

Sakata et Ball speed

al. [43]

3) Throwing velocity (standing) (ND)

4) Throwing velocity (jumping) (ND)

5) Serve accuracy

D, dominant shoulder; ND, non-dominant shoulder; ER, external rotation; HA, horizontal adduction; IR, internal rotation; PT, peak torque; ROM, range of motion; TW, total work

${ }^{\wedge}$ Deficits defined as the difference between dominant and non-dominant limbs

Table 5

Effectiveness of each outcome measure categorised by performance category

\begin{tabular}{|lllll|}
\hline $\begin{array}{l}\text { Performance } \\
\text { category }\end{array}$ & $\begin{array}{l}\text { No. of significantly } \\
\text { improved performance } \\
\text { outcome measures }\end{array}$ & $\begin{array}{l}\text { No. of not significantly } \\
\text { improved performance } \\
\text { outcome measures }\end{array}$ & Total & $\begin{array}{l}\text { Effectiveness } \\
\text { (\%) }\end{array}$ \\
\hline Strength & 7 & 16 & 23 & 30.4 \\
\hline Mobility & 2 & 5 & 7 & 28.6 \\
\hline $\begin{array}{l}\text { Sport- } \\
\text { specific }\end{array}$ & 2 & 7 & 9 & 22.2 \\
\hline
\end{tabular}

\section{Strength-based performance outcome measures}


A total of 23 strength-based performance outcome measures were identified across three IPPs, with 18 unique outcome measures [44-46]. Performance outcome measures of strength repeatedly measured across the studies were dominant limb isokinetic concentric (conventional) external rotation/internal rotation (ER/IR) strength balance ratio at $60 \%$ s (2 studies), dominant limb isokinetic concentric IR peak torque (PT) at $60 \%$ s (2 studies), dominant limb isokinetic concentric ER PT at $60 \%$ s (2 studies), dominant limb isokinetic eccentric ER PT at $240^{\circ}$ /s (2 studies), and dominant limb isokinetic functional ER/IR ratio at $240^{\circ} / \mathrm{s}$ ( 2 studies).

Only one study [46] reported significant improvements in the intervention group as compared to the control group for dominant limb isokinetic conventional ER/IR strength balance ratio at $60 \%$ s. The same study also reported significant improvements for non-dominant limb isokinetic concentric IR and ER PT at $60 \%$, and non-dominant limb isokinetic eccentric ER PT at $240 \%$ s. Other strength-based performance outcome measures that significantly improved compared to controls were isokinetic eccentric shoulder ER strength at $90 \%$ s [44], isokinetic concentric ER total work at $60 \%$ s [46], and isokinetic concentric IR average power at $240 \%$ s [45].

\section{Mobility-based performance outcome measures}

Seven mobility-based performance outcome measures were investigated across two IPPs, with six unique outcome measures $[38,43]$. Total range of motion (ROM) in the dominant shoulder was the only mobility performance outcome measure evaluated across both studies. The remaining five unique outcome measures were all investigated from the same study [43].

Compared to controls, the intervention groups in both studies did not demonstrate significant improvements for total ROM [38, 43]. In contrast, significant improvements were observed for shoulder horizontal adduction ROM deficits and thoracic kyphosis angle [43].

\section{Sport-specific performance outcome measures}

A total of nine sport-specific performance outcome measures were investigated, of which, eight were related to ball speed and one to serve accuracy.

Eight performance outcome measures related to ball speed, otherwise labelled as serve velocity, throwing velocity, and throwing speed, were examined across four IPPs for tennis [38], handball [45, 46], and baseball [43]. The magnitude of a ball velocity represents the speed of the ball, which is the variable of interest and therefore represent the same measure in this review. Amongst these eight measures, four unique performance outcome measures were identified.

Ball speed as a performance outcome was commonly measured across the studies as dominant limb ball speed during standing throws (4 studies) and dominant limb ball speed during jumping throws ( 2 studies). Tennis serve [38] was analysed as a standing throw as no run-up is involved in the execution of the motion. Dominant limb ball speed during standing throws showed significant improvements in two 
studies [38, 43]. However, no significant improvements were observed for dominant limb ball speed during jumping throws $[45,46]$.

Only one study investigated serve accuracy as a performance outcome measure [38]. No significant improvement compared to the controls was reported.

\section{Discussion}

The primary objective of this systematic review was to evaluate the effectiveness of existing upper extremity IPPs on modifying performance outcome measures in overhead youth athletes. The various performance outcome measures identified in the studies were classified into three categories - strength, mobility, and sport-specific performance measures. Sport-specific performance measures were further categorised into measures of ball speed and serve accuracy. Strength-based performance outcome measures made up the majority of the identified performance outcome measures with 23 measurements, followed by sport-specific measures with nine (eight related to ball speed and one related to serve accuracy), and mobility-based measures with seven measurements. In terms of the effectiveness on upper extremity performance, outcome measures of strength significantly improved at a rate of $30.4 \%$. This was followed by mobility-based outcomes at $28.6 \%$, and sport-specific outcomes at $22.2 \%$, suggesting that upper extremity IPPs have modest effects on the performance outcome measures.

The secondary purpose was to identify and categorize the training components targeted by the existing IPPs. The three training categories identified were strength, mobility, and plyometrics. Strength was the most popular targeted training component across the studies, with its associated performance outcome measures being the most widely investigated.

\section{Strength intervention-based performance outcome measures}

Studies investigating the biomechanics of the overhead motion have documented forces of large magnitude in the shoulder complex. [18, 24]. To illustrate, elite baseball pitchers can generate distractive forces at the shoulder equivalent to the thrower's body weight when performing a baseball pitch [48]. To prevent shoulder joint distraction, compressive forces of the same magnitude needs to be generated, which requires eccentric contraction of the scapular and elbow muscles [49]. Failure of these muscles to sustain the repeated large magnitudes of eccentric contraction due to poor external rotator eccentric strength, can lead to overuse injuries [49]. This has spurred the interest in shoulder strength measures, specifically shoulder strength balance ratio of external to internal rotators.

The exercises for the development of shoulder rotator strength were generally similar across the programs, with the shoulder either abducted to $90^{\circ}$ with elbow flexed at $90^{\circ}$, or remain in neutral position with elbow flexed at $90^{\circ}$ and tucked against the side of the trunk. Both positions facilitate shoulder external and internal rotation. To develop strength, the shoulder was moved through the rotational range 
of motion while using some forms of resistance, such as resistance bands $[38,45,46]$ and cricket balls [44].

The overall effectiveness of the IPPs on strength performance was only modest at $30.4 \%$. This could be due to the short duration over which the IPPs were conducted. Two studies conducted their program over six weeks with a total of 18 sessions $[45,46]$, while one conducted their warm-up exercise program over eight weeks with a total of 16 sessions [44]. A recent systematic review and meta-analysis of resistance training among youth athletes showed that strength improvement in youth athletes was more pronounced in programs with training periods of more than 23 weeks, with no significant differences between training frequencies of 1, 2, or 3 times per week [50]. Therefore, the lack of significant improvements in strength-based performance outcome measures as compared to controls in the studies included in this review could be attributed to the sub-optimal training intervention period. Future studies investigating strength-based performance outcome measures among youth athletes should consider a duration of 23 weeks or longer to enhance the methodological validity of strength measurements.

The non-significant improvements in strength-based performance outcome measures could also be due to differences between isokinetic testing speeds and the speeds at which the exercises were performed in real-time. These differences could have impacted the results of the strength-based performance outcome measures based on the training principle of specificity [44]. Moreover, despite performing the same action (e.g., concentric ER), different testing speeds were utilized for the isokinetic strength tests across studies (e.g., $60 \%$ s and $240 \%$ s), resulting in methodological differences. To improve understanding and allow a valid comparison across studies, a standardised measurement protocol for isokinetic strength testing is required, in addition to employing testing speeds comparable to the speed of the exercises performed in real-time.

Among the seven significantly improved strength performance outcome measures, four were observed in the non-dominant limb of the athletes. It is likely that the non-dominant limbs of overhead athletes were relatively less trained and hence had a larger potential for muscular strength gain [46], in contrast to the dominant limbs which are likely to be relatively well-trained due to repeated use. Although reasonable, this hypothesis should be addressed by future studies reporting absolute strength measures for both dominant and non-dominant limbs before and after the IPP to determine the absolute strength gain.

All seven of the strength-based performance outcome measures that significantly improved compared to controls were isokinetic measures. Although isokinetic strength measures are the gold-standard for measuring shoulder strength, the equipment and procedures required in isokinetic strength measurements are expensive and cannot be used for on-field measurements [51]. In contrast, the handheld dynamometer (HHD) used for isometric strength measurements is low-cost, portable, and suitable for field use. Additionally, the HHD has also demonstrated high intra- and inter-rater reliability for isometric shoulder strength measurements, making it a suitable alternative to an isokinetic device [51, 52]. Furthermore, using HHD for strength measurements may also lead to a greater protocol consistency across studies thus enabling researchers to draw valid conclusions while comparing the studies. This 
provides a reasonable basis for the future studies to investigate isometric strength measures utilizing HHDs and enable practitioners like coaches and trainers to use the device on-field as they may not always have access to specialised facilities and expert manpower.

\section{Mobility-based performance outcome measures and its effectiveness}

Mobility measures related to the shoulder (i.e., ER ROM, IR ROM, horizontal adduction ROM, and total ROM) were found to be of popular interest. These measurements have also been investigated in other studies [53-56], further substantiating the interest in mobility measures amongst researchers. This stems from the adaptations observed in the dominant shoulder of an overhead athlete, where a decrease in IR ROM and an increase in ER ROM were documented, while maintaining total ROM [57]. These adaptations may constitute risk factors for shoulder overuse injury among overhead athletes of all ages [24,58-60]. In a meta-analysis to determine the risk of upper extremity injury due to glenohumeral internal rotation deficit (GIRD) in overhead athletes [59], shoulders with GIRD were at a higher risk of sustaining an upper extremity injury, with a mean difference of $3.11^{\circ}(p=0.06,95 \% \mathrm{Cl}[-0.13-6.36])$ between injured and uninjured athletes. The interest in ROM measurements also stems from the importance of having adequate shoulder mobility for throwing performance, with greater shoulder mobility allowing a greater arc of motion through which the throwing arm can accelerate to produce high velocities at ball release or ball contact $[25,56,61]$. Therefore, efforts to restore or maintain a healthy range of motion in the shoulder for injury prevention and performance enhancement have become a popular area of investigation among sports injury researchers.

The only two performance outcome measures (out of seven) that significantly improved as compared to controls (28.6\%) were thoracic kyphosis angle and shoulder horizontal adduction ROM deficits. Both measures were observed from the study where the mYKB-9 program was performed [43]. The significant improvement in thoracic kyphosis angle as compared to controls [43] could be attributed to the dynamic thoracic mobility exercises (i.e., cat and dog exercise and trunk rotation exercise) performed in the mYKB9 program. Thoracic kyphosis is considered as a risk factor for shoulder injuries in overhead athletes as the flexed thoracic spine results in a protracting scapula which alters shoulder mechanics [62]. With the nature of overhead sports requiring repeated forceful overhead motions, the repeated execution of improper shoulder mechanics is likely to increase the risk of shoulder injury.

Shoulder problems of horizontal adduction ROM deficits and GIRD are closely related. They occur as a result of posterior shoulder tightness, an adaptation from the repetitive throwing or striking actions required in overhead sports [57, 63, 64]. In relation to this, performing the posterior shoulder stretch (as part of the mYKB-9 program) should theoretically lead to reduced posterior shoulder tightness and hence improved shoulder horizontal adduction ROM deficits and GIRD. However, compared to controls, only the performance outcome measure of shoulder horizontal adduction ROM deficits saw significant improvements [43]. No significant improvement for GIRD was observed among the same participants 
[43]. This is surprising since both shoulder problems are considered as an outcome of posterior shoulder tightness $[65,66]$.

The non-significant improvements in IR ROM reported in the mYKB-9 program [43] could be attributed to the way the posterior shoulder stretch was carried out and the lack of a stretch specifically targeting IR ROM (i.e., sleeper stretch). Generally, the cross-body stretch (also known as the horizontal adduction stretch) and the sleeper stretch are recommended to reduce posterior shoulder tightness and improve horizontal adduction and IR ROM $[63,66,67]$. However, neither of the stretches were included in the mYKB-9 [43]. Instead of the usual individually-performed cross-body stretch [63] where the non-stretching arm pushes the stretching arm into horizontal adduction while in a standing position (open kinetic chain), the posterior shoulder stretch in the mYKB-9 [43] involved the athlete using their body weight to pull the shoulder into a stretch while being on all-fours (closed kinetic chain). The difference in these stretching techniques could have affected the structure and ability of the posterior shoulder stretch to improve shoulder IR ROM. Additionally, the lack of a stretch specifically targeting IR ROM in the MYKB-9 [43] is in contrast to the original YKB-9, where the sleeper stretch was included and which yielded significant improvements in shoulder IR ROM deficits [47]. Therefore, it is likely that inclusion of sleeper stretch in the mYKB-9 could have led to significant improvements in IR ROM deficits.

Modifications to the traditional cross-body stretch and sleeper stretch have been recently suggested, which stabilises the scapula minimizing the symptoms of pain and increasing the stretch [63]. These modified stretches were effective at increasing shoulder IR and horizontal adduction ROM among college baseball players [68]. Future upper extremity injury prevention interventions that aim to improve IR ROM and shoulder horizontal adduction ROM might benefit from utilizing the modified sleeper stretch and cross-body stretch proposed by Wilk and colleagues [63].

\section{Sport-specific performance outcome measures and its effectiveness}

Only two sport-specific performance outcome measures were assessed in the identified studies: serve accuracy and ball speed. Serve accuracy was explicitly evaluated in only one study [38]. However, during the measurements for ball speed, the athletes had to aim their serves or throws towards a target to attain accurate measures $[43,45,46]$. Therefore, accuracy was indirectly incorporated during the measurements of ball speed. Nevertheless, explicitly assessing throwing or serving accuracy as a performance outcome measure would only serve to further enhance the specificity of the performance assessment of an athlete.

Fernandez-Fernandez et al [38] assessed both serve velocity (i.e., ball speed) and serve accuracy in their study on male junior tennis players. Only the serve velocity significantly improved as compared to controls while the mean differences of change in serve accuracy scores between the two groups were non-significant. However, both experimental and control groups showed improvements in serve accuracy scores from pre-test to post-test, albeit non-significant. These improvements in serve accuracy likely occurred due to the regular tennis training sessions attended by all participants of the study. Overall, this implies that despite serving at significantly higher velocities than controls, the athletes in the 
experimental group were still able to maintain their serve accuracy. Being able to execute high-speed serves while maintaining accuracy is critical to an athlete's performance. Hence, the 6-week strength training program for youth tennis players can be considered to be effective despite the non-significant improvements in serve accuracy.

Significant improvements in ball speed as compared to controls was also observed in the study where the mYKB-9 was implemented [43]. The mYKB-9 did not include any strength-based or plyometric exercises. It only consisted of stretching, dynamic mobility, and lower extremity balance training exercises that were based on the previous YKB-9 prevention program [47]. Therefore, the significant improvement in ball speed, despite the lack of strength or plyometric exercises could be attributed to the previously identified significant improvements in shoulder horizontal adduction ROM deficits and thoracic kyphosis angle [43]. Improvements in these mobility measures likely corrected the deficits that contributed to poor activation and coordination of the muscles involved in the overhead motion kinetic chain $[49,69]$. Hence, we suggest that future IPPs target improving upper extremity mobility, as the improvements are likely to crossover and also enhance sport-specific performance outcome measures (e.g., ball speed).

\section{Implications}

Future studies targeting shoulder strength performance outcome measures in youth athletes should conduct their program over a duration of at least 23 weeks. If isokinetic strength measures are utilized, standardised measurement protocols should be established to allow comparisons across studies. To achieve a higher ecological validity and to address the training principle of specificity, testing speeds should be similar to the speeds at which the exercises are performed in real-time. Additionally, with the $\mathrm{HHD}$ offering a low-cost, portable, simple to use and reliable alternative, more research employing isometric strength measures using this device should be considered.

Further research targeting upper extremity mobility and its performance outcome measures in the youth overhead sport population is required. As the dynamic trunk mobility exercise [43] and modified sleeper and cross-body stretches have been reported to be effective exercises [68], future upper extremity injury prevention interventions should include these exercises in their IPPs to improve measures of mobility.

More research on the effects of upper extremity IPPs on sport-specific performance outcome measure of serve/throwing accuracy is needed. Future upper extremity IPPs could focus more on mobility-based exercises, as improvements in upper extremity mobility are likely to lead to consequent improvements in sport-specific performance (i.e., ball speed). More upper extremity sport-specific plyometric-based exercises could also be included in upper extremity IPPs.

Lastly, while blinding of all therapists (who administered the therapy) is not feasible due to the nature of the research, blinding the participants and assessors (who measured at least one key outcome) are possible. A suggestion would be to implement a sham exercise program for participants in the control group and ensuring that the assessors and therapists involved in the study are unrelated.

\section{Limitations}


This systematic review is not without limitations. Language bias is possible as only articles published in the English language were included. Due to differences in measurement protocols, a wide variety of performance outcome measures were found across the included studies. To address this limitation, similar performance outcome measures were categorised into a common category. For example, isokinetic concentric ER PT at $60 \%$ and isokinetic eccentric ER strength at $90 \%$ s were both grouped into the category of strength performance outcome measures. This allowed for the evaluation of overall effectiveness of the IPPs on certain categories of performance outcome measures. However, the subjective classification of the performance outcome measures into categories may have introduced a bias. Similarly, the classification of exercises into training components was also subjective. A small number of eligible studies, variability in the number of exercises, repetitions, sets, frequency and duration of intervention across the IPPs precluded the conduct of meta-analysis, which would have increased the strength of conclusions from this systematic review. Compliance to the prevention programs was not analysed in this systematic review, which could have further contributed to understanding the effects of existing upper extremity IPPs on upper extremity performance outcome measures in overhead youth athletes.

\section{Conclusion}

There is a relative dearth of methodologically robust upper extremity IPPs in overhead youth athletes. The training components of the identified upper extremity IPPs were strength, mobility, and plyometrics, with strength being the most common training component. Performance outcome measures identified from IPPs in the five studies were categorised into three categories of strength, mobility, and sport-specific measures. Strength-based performance outcome measures formed the majority of the outcome measures evaluated and had the highest overall effectiveness rate, followed by mobility and sportspecific performance measures. Future IPPs should include training components of strength, mobility, and plyometrics, as they have been observed to improve performance outcome measures of strength, mobility, and sport-specific measures, albeit to modest degrees. Protocols for the sport-specific exercises, volume, repetitions, sets, and overall program duration of an IPP also needs to be established to allow for future quantitative statistical analysis. Standardised measurement protocols should be established especially for strength measures to ensure consistency in the reporting of intervention components and performance outcome measures.

\section{Abbreviations}

ER: external rotation; GIRD: Glenohumeral Internal Rotation Deficit; HHD: hand-held dynamometer; IR: internal rotation; IPP: injury prevention program; NOS: Newcastle-Ottawa Scale; mYKB-9: modified Yokohama Baseball-9; PEDro: Physiotherapy Evidence Database; PICOS; Population, Intervention, Comparison, Outcome, Study design; PRISMA: Preferred Reporting Items for Systematic Reviews and Meta-Analyses; PT: Peak Torque; RCT: randomized controlled trial; RIO: High School Reporting Information Online; ROM: range of motion; YKB-9: Yokohama Baseball-9 


\section{Declarations}

\section{Ethics approval and consent to participate}

No patients or participants were directly involved in the research. The present study used public data from the existing studies published in the academic literature to perform a systematic review. The University Institutional Review Board exempts such studies from the need of any review.

\section{Consent for publication}

Not applicable.

\section{Availability of data and materials}

All data used in the present study were retrieved from the publications used in the systematic review and are publicly available.

\section{Competing interests}

The authors declare that they have no competing interests.

\section{Funding}

No sources of funding were used in the conduct of this study

\section{Authors' contributions}

$\mathrm{RL}$ contributed to the conception and design of the review. Both $\mathrm{RL}$ and $\mathrm{SW}$ conducted the analysis and interpretation of the data, the drafting and revising of the manuscript, agreed on the final draft, and take responsibility for the integrity and accuracy of the works.

\section{Acknowledgements}

Not applicable.

\section{References}

1. NFHS: 2018-19 High school athletics participation

survey. https://www.nfhs.org/media/1020412/2018-19_participation_survey.pdf (2019). Accessed 5 July 
2020.

2. Bailey R. Physical education and sport in schools: A review of benefits and outcomes. J Sch Health. 2006;76(8):397-401. doi: 10.1111/j.1746-1561.2006.00132.x.

3. Difiori J. Overuse injury of the physis: a "growing" problem. Clin J Sport Med. 2010;20(5):336-7. doi: 10.1097/JSM.0b013e3181ebb55d.

4. International Olympic Committee: What is YOG? https://www.olympic.org/news/what-is-yog (2020). Accessed 5 October 2020.

5. Sport Singapore: Another first for the Asian Youth Games - Official Website Launched. https://www.sportsingapore.gov.sg/newsroom/media-releases/2009/5/another-first-for-theasian-youth-games-official-website-launched (2009). Accessed 5 October 2020.

6. Adirim TA, Cheng TL. Overview of injuries in the young athlete. Sports Med. 2003;33(1):75-81. doi: 10.2165/00007256-200333010-00006.

7. Cassas KJ, Cassettari-Wayhs A. Childhood and adolescent sports-related overuse injuries. Am Fam Physician. 2006;73(6):1014-22.

8. Zaremski JL, Zeppieri G, Tripp BL. Sport specialization and overuse injuries in adolescent throwing athletes: a narrative review. J Athl Train. 2019;54(10):1030-9. doi: 10.4085/1062-6050-333-18.

9. Caine D, Maffulli N. Epidemiology of children's individual sports injuries. An important area of medicine and sport science research. Med Sport Sci. 2005;48:1-7. doi: 10.1159/000084274.

10. Carder SL, Giusti NE, Vopat LM, Tarakemeh A, Baker J, Vopat BG, et al. The concept of sport sampling versus sport specialization: preventing youth athlete injury: a systematic review and meta-analysis. Am J Sports Med. 2020;48(11):2850-7. doi: 10.1177/0363546519899380.

11. Bell DR, Post EG, Biese K, Bay C, Valovich McLeod T. Sport specialization and risk of overuse injuries: a systematic review with meta-analysis. Pediatrics. 2018;142(3). doi: 10.1542/peds.2018-0657.

12. Jayanthi NA, Post EG, Laury TC, Fabricant PD. Health Consequences of Youth Sport Specialization. J Athl Train. 2019;54(10):1040-9. doi: 10.4085/1062-6050-380-18.

13. DiFiori J, Benjamin H, Brenner J, Gregory A, Jayanthi N, Landry G, et al. Overuse injuries and burnout in youth sports: a position statement from the American Medical Society for Sports Medicine. Br J Sports Med. 2014;48(4):287-8. doi: 10.1136/bjsports-2013-093299.

14. Aasheim C, Stavenes H, Andersson SH, Engbretsen L, Clarsen B. Prevalence and burden of overuse injuries in elite junior handball. BMJ Open SEM. 2018;4(1):e000391. doi: 10.1136/bmjsem-2018-000391. 
15. Kraan RBJ, Nobel Dd, Eygendaal D, Daams JG, Kuijer PPFM, Maas M. Incidence, prevalence, and risk factors for elbow and shoulder overuse injuries in youth athletes: A systematic review. Transl Sports Med. 2019;2(4):186-95. doi: 10.1002/tsm2.82.

16. Magra M, Caine D, Maffulli N. A review of epidemiology of paediatric elbow injuries in sports. Sports Med. 2007;37(8):717-35. doi: 10.2165/00007256-200737080-00005.

17. Lintner D, Noonan TJ, Kibler WB. Injury patterns and biomechanics of the athlete's shoulder. Clin Sports Med. 2008;27(4):527-51. doi: 10.1016/j.csm.2008.07.007.

18. Braun S, Kokmeyer D, Millett PJ. Shoulder injuries in the throwing athlete. J Bone Joint Surg. 2009;91(4):966-78. doi: 10.2106/JBJS.H.01341.

19. Wilk KE, Obma P, Simpson CD, Cain EL, Dugas JR, Andrews JR. Shoulder injuries in the overhead athlete. J Orthop Sports Phys Ther. 2009;39(2):38-54. doi: 10.2519/jospt.2009.2929.

20. Asker $M$, Brooke HL, Waldén $M$, Tranaeus $U$, Johansson F, Skillgate $E$, et al. Risk factors for, and prevention of, shoulder injuries in overhead sports: a systematic review with best-evidence synthesis. $\mathrm{Br} \mathrm{J}$ Sports Med. 2018;52(20):1312-9. doi: 10.1136/bjsports-2017-098254.

21. Frisch KE, Clark J, Hanson C, Fagerness C, Conway A, Hoogendoorn L. High prevalence of nontraumatic shoulder pain in a regional sample of female high school volleyball athletes. Orthop $\mathrm{J}$ Sports Med. 2017;5(6). doi: 10.1177/2325967117712236.

22. Roos KG, Marshall SW, Kerr ZY, Golightly YM, Kucera KL, Myers JB, et al. Epidemiology of overuse injuries in collegiate and high school athletics in the United States. Am J Sports Med. 2015;43(7):1790-7. doi: 10.1177/0363546515580790.

23. Frisch A, Croisier J-L, Urhausen A, Seil R, Theisen D. Injuries, risk factors and prevention initiatives in youth sport. Br Med Bull. 2009;92(1):95-121. doi: 10.1093/bmb/ldp034.

24. Lin DJ, Wong TT, Kazam JK. Shoulder injuries in the overhead-throwing athlete: Epidemiology, mechanisms of injury, and imaging findings. Radiology. 2018;286(2):370-87. doi: 10.1148/radiol.2017170481.

25. Fleisig GS, Barrentine SW, Escamilla RF, Andrews JR. Biomechanics of overhand throwing with implications for injuries. Sports Med. 1996;21(6):421-37. doi: 10.2165/00007256-199621060-00004.

26. Olsen O-E, Myklebust G, Engebretsen L, Holme I, Bahr R. Exercises to prevent lower limb injuries in youth sports: cluster randomised controlled trial. BMJ. 2005;330(7489):449. doi:

10.1136/bmj.38330.632801.8F.

27. Emery CA, Rose MS, McAllister JR, Meeuwisse WH. A prevention strategy to reduce the incidence of injury in high school basketball: a cluster randomized controlled trial. Clin J Sport Med. 2007;17(1):17-24. 
doi: 10.1097/JSM.0b013e31802e9c05.

28. Soomro N, Sanders R, Hackett D, Hubka T, Ebrahimi S, Freeston J, et al. The efficacy of injury prevention programs in adolescent team sports: a meta-analysis. Am J Sports Med. 2016;44(9):2415-24. doi: $10.1177 / 0363546515618372$.

29. Rössler R, Donath L, Verhagen E, Junge A, Schweizer T, Faude O. Exercise-based injury prevention in child and adolescent sport: a systematic review and meta-analysis. Sports Med. 2014;44(12):1733-48. doi: 10.1007/s40279-014-0234-2.

30. Emery CA, Roy T-O, Whittaker JL, Nettel-Aguirre A, van Mechelen W. Neuromuscular training injury prevention strategies in youth sport: a systematic review and meta-analysis. Br J Sports Med. 2015;49(13):865-70. doi: 10.1136/bjsports-2015-094639.

31. Bahr R, Krosshaug T. Understanding injury mechanisms: a key component of preventing injuries in sport. Br J Sports Med. 2005;39(6):324-9. doi: 10.1136/bjsm.2005.018341.

32. Leppänen M, Aaltonen S, Parkkari J, Heinonen A, Kujala UM. Interventions to prevent sports related injuries: a systematic review and meta-analysis of randomised controlled trials. Sports Med. 2014;44(4):473-86. doi: 10.1007/s40279-013-0136-8.

33. Hanlon C, Krzak JJ, Prodoehl J, Hall KD. Effect of injury prevention programs on lower extremity performance in youth athletes: a systematic review. Sports Health. 2020;12(1):12-22. doi: $10.1177 / 1941738119861117$.

34. van Mechelen W, Hlobil H, Kemper HC. Incidence, severity, aetiology and prevention of sports injuries. A review of concepts. Sports Med. 1992;14(2):82-99. doi: 10.2165/00007256-199214020-00002.

35. Faude O, Rössler R, Petushek EJ, Roth R, Zahner L, Donath L. Neuromuscular adaptations to multimodal injury prevention programs in youth sports: a systematic review with meta-analysis of randomized controlled trials. Front Physiol. 2017;8:791. doi: 10.3389/fphys.2017.00791.

36. Moher D, Liberati A, Tetzlaff J, Altman DG, Group P. Preferred reporting items for systematic reviews and meta-analyses: the PRISMA statement. BMJ. 2009;339:b2535. doi: 10.1136/bmj.b2535.

37. Crossley KM, Patterson BE, Culvenor AG, Bruder AM, Mosler AB, Mentiplay BF. Making football safer for women: a systematic review and meta-analysis of injury prevention programmes in 11773 female football (soccer) players. Br J Sports Med. 2020;54(18):1089-98. doi: 10.1136/bjsports-2019-101587.

38. Fernandez-Fernandez J, Ellenbecker T, Sanz-Rivas D, Ulbricht A, Ferrautia A. Effects of a 6-week junior tennis conditioning program on service velocity. J Sports Sci Med. 2013;12(2):232-9.

39. Plummer A, Mugele H, Steffen K, Stoll J, Mayer F, Müller J. General versus sports-specific injury prevention programs in athletes: A systematic review on the effects on performance. PLoS One. 
2019;14(8):e0221346. doi: 10.1371/journal.pone.0221346.

40. de Morton NA. The PEDro scale is a valid measure of the methodological quality of clinical trials: a demographic study. Aust J Physiother. 2009;55(2):129-33. doi: 10.1016/s0004-9514(09)70043-1.

41. Wells GA, Shea B, O'Connell D, Peterson J, Welch V, Losos M, et al.: The Newcastle-Ottawa Scale (NOS) for assessing the quality of nonrandomised studies in meta-

analyses. http://www.ohri.ca/programs/clinical_epidemiology/oxford.asp (2021). Accessed 10 December 2020.

42. Armijo-Olivo S, da Costa BR, Cummings GG, Ha C, Fuentes J, Saltaji H, et al. PEDro or Cochrane to assess the quality of clinical trials? a meta-epidemiological study. PLoS One. 2015;10(7):e0132634. doi: $10.1371 /$ journal.pone.0132634.

43. Sakata J, Nakamura E, Suzuki T, Suzukawa M, Akeda M, Yamazaki T, et al. Throwing injuries in youth baseball players: can a prevention program help? a randomized controlled trial. Am J Sports Med. 2019;47(11):2709-16. doi: 10.1177/0363546519861378.

44. Forrest MRL, Hebert JJ, Scott BR, Dempsey AR. Exercise-based injury prevention for community-level adolescent cricket pace bowlers: A cluster-randomised controlled trial. J Sci Med Sport. 2020;23(5):47580. doi: 10.1016/j.jsams.2019.12.009.

45. Mascarin NC, de Lira CAB, Vancini RL, de Castro Pochini A, da Silva AC, Dos Santos Andrade M. Strength training using elastic bands: improvement of muscle power and throwing performance in young female handball players. J Sport Rehabil. 2017;26(3):245-52. doi: 10.1123/jsr.2015-0153.

46. Mascarin NC, de Lira CAB, Vancini RL, da Silva AC, Andrade MS. The effects of preventive rubber band training on shoulder joint imbalance and throwing performance in handball players: A randomized and prospective study. J Bodyw Mov Ther. 2017;21(4):1017-23. doi: 10.1016/j.jbmt.2017.01.003.

47. Sakata J, Nakamura E, Suzuki T, Suzukawa M, Akaike A, Shimizu K, et al. Efficacy of a prevention program for medial elbow injuries in youth baseball players. Am J Sports Med. 2018;46(2):460-9. doi: $10.1177 / 0363546517738003$.

48. Werner SL, Gill TJ, Murray TA, Cook TD, Hawkins RJ. Relationships between throwing mechanics and shoulder distraction in professional baseball pitchers. Am J Sports Med. 2001;29(3):354-8. doi: $10.1177 / 03635465010290031701$.

49. Weber AE, Kontaxis A, O'Brien SJ, Bedi A. The biomechanics of throwing: simplified and cogent. Sports Med Arthrosc Rev. 2014;22(2):72-9. doi: 10.1097/jsa.0000000000000019.

50. Lesinski M, Prieske O, Granacher U. Effects and dose-response relationships of resistance training on physical performance in youth athletes: a systematic review and meta-analysis. Br J Sports Med. 2016;50(13):781-95. doi: 10.1136/bjsports-2015-095497. 
51. Cools AM, Johansson FR, Borms D, Maenhout A. Prevention of shoulder injuries in overhead athletes: a science-based approach. Braz J Phys Ther. 2015;19(5):331-9. doi: 10.1590/bjpt-rbf.2014.0109.

52. Fredriksen $\mathrm{H}$, Cools A, Bahr R, Myklebust G. Does an effective shoulder injury prevention program affect risk factors in handball? A randomized controlled study. Scand J Med Sci Sports. 2020;30(8):142333. doi: $10.1111 / \mathrm{sms} .13674$.

53. Gulpinar D, Tekeli Ozer S, Yesilyaprak SS. Effects of rigid and kinesio taping on shoulder rotation motions, posterior shoulder tightness, and posture in overhead athletes: a randomized controlled trial. J Sport Rehabil. 2019;28(3):256-65. doi: 10.1123/jsr.2017-0047.

54. Kibler WB, Chandler TJ. Range of motion in junior tennis players participating in an injury risk modification program. J Sci Med Sport. 2003;6(1):51-62. doi: 10.1016/s1440-2440(03)80008-7.

55. Oranchuk DJ, Ecsedy EN, Robinson TL. Effects of a sport-specific upper-body resistance-band training program on overhead throwing velocity and glenohumeral joint range of motion. J Strength Cond Res. 2020. doi: $10.1519 /$ JSC.0000000000003303.

56. Yang WW, Liu YC, Lu LC, Chang HY, Chou PP, Liu C. Performance enhancement among adolescent players after 10 weeks of pitching training with appropriate baseball weights. J Strength Cond Res. 2013;27(12):3245-51. doi: 10.1519/JSC.0b013e31828ddfeb.

57. Borsa PA, Laudner KG, Sauers EL. Mobility and stability adaptations in the shoulder of the overhead athlete: a theoretical and evidence-based perspective. Sports Med. 2008;38(1):17-36. doi:

10.2165/00007256-200838010-00003.

58. Shanley E, Rauh MJ, Michener LA, Ellenbecker TS, Garrison JC, Thigpen CA. Shoulder range of motion measures as risk factors for shoulder and elbow injuries in high school softball and baseball players. Am J Sports Med. 2011;39(9):1997-2006. doi: 10.1177/0363546511408876.

59. Keller RA, De Giacomo AF, Neumann JA, Limpisvasti O, Tibone JE. Glenohumeral internal rotation deficit and risk of upper extremity injury in overhead athletes: A meta-analysis and systematic review. Sports Health. 2018;10(2):125-32. doi: 10.1177/1941738118756577.

60. Astolfi MM, Struminger AH, Royer TD, Kaminski TW, Swanik CB. Adaptations of the shoulder to overhead throwing in youth athletes. J Athl Train. 2015;50(7):726-32. doi: 10.4085/1062-6040-50.1.14.

61. Roach NT, Lieberman DE, Gill Iv TJ, Palmer WE, Gill lii TJ. The effect of humeral torsion on rotational range of motion in the shoulder and throwing performance. J Anat. 2012;220(3):293-301. doi:

10.1111/j.1469-7580.2011.01464.x.

62. Kibler WB, Ludewig PM, McClure PW, Michener LA, Bak K, Sciascia AD. Clinical implications of scapular dyskinesis in shoulder injury: the 2013 consensus statement from the 'scapular summit'. Br J Sports Med. 2013;47(14):877-85. doi: 10.1136/bjsports-2013-092425.

Page 32/34 
63. Wilk KE, Hooks TR, Macrina LC. The modified sleeper stretch and modified cross-body stretch to increase shoulder internal rotation range of motion in the overhead throwing athlete. J Orthop Sports Phys Ther. 2013;43(12):891-4. doi: 10.2519/jospt.2013.4990.

64. Mine K, Nakayama T, Milanese S, Grimmer K. Effectiveness of stretching on posterior shoulder tightness and glenohumeral internal rotation deficit: a systematic review of randomised controlled trials. J Sport Rehabil. 2016;26(4):294-305. doi: 10.1123/jsr.2015-0172.

65. Shanley E, Thigpen C. Throwing injuries in the adolescent athlete. Int J Sports Phys Ther. 2013;8(5):630-40.

66. Cools AM, Johansson FR, Cagnie B, Cambier DC, Witvrouw EE. Stretching the posterior shoulder structures in subjects with internal rotation deficit: comparison of two stretching techniques. Shoulder Elbow. 2012;4(1):56-63. doi: 10.1111/j.1758-5740.2011.00159.x.

67. Tyler TF, Nicholas SJ, Lee SJ, Mullaney M, McHugh MP. Correction of posterior shoulder tightness is associated with symptom resolution in patients with internal impingement. Am J Sports Med. 2010;38(1):114-9. doi: 10.1177/0363546509346050.

68. Yamauchi T, Hasegawa S, Nakamura M, Nishishita S, Yanase K, Fujita K, et al. Effects of two stretching methods on shoulder range of motion and muscle stiffness in baseball players with posterior shoulder tightness: a randomized controlled trial. J Shoulder Elbow Surg. 2016;25(9):1395-403. doi: 10.1016/j.jse.2016.04.025.

69. Eckenrode BJ, Kelley MJ, Kelly JDt. Anatomic and biomechanical fundamentals of the thrower shoulder. Sports Med Arthrosc Rev. 2012;20(1):2-10. doi: 10.1097/JSA.0b013e3182471f03.

70. Forrest MRL, Scott BR, Hebert JJ, Dempsey AR. Injury prevention strategies for adolescent cricket pace bowlers. Sports Med. 2018;48(11):2449-61. doi: 10.1007/s40279-018-0981-6.

\section{Figures}




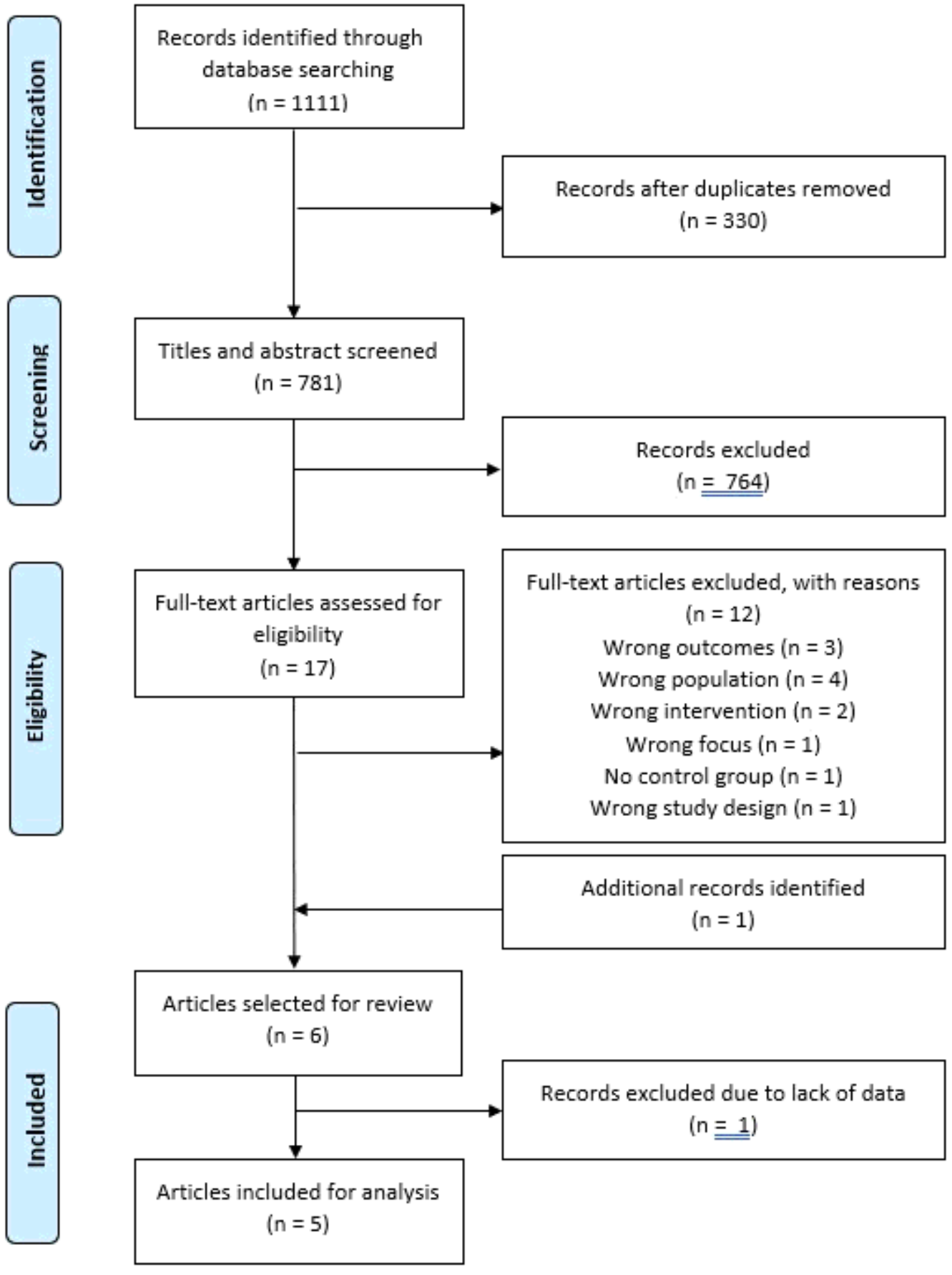

\section{Figure 1}

Study identification, screening, and exclusion pathway 